\title{
Teorías de la interpretación jurídica y teorías del significado
}

\author{
Damiano Canale* \\ Universidad "L. Bocconi", Milán \\ Traducción de Magdalena Ana Rosso**
}

\section{1. ¿Una teoría de la interpretación jurídica presupone una teoría del significado?}

En "El escepticismo ante las reglas replanteado", Riccardo Guastini presenta una síntesis de su investigación en el campo de la teoría de la interpretación jurídica. Una investigación cuyos resultados, objeto con el correr de los años de continuos refinamientos, ofrecen al jurista y al filósofo del derecho innumerables puntos de reflexión.

En la primera parte del ensayo, Guastini distingue los diferentes significados que asume el término "interpretación" en el discurso de los juristas y señala bajo cuáles aspectos la interpretación constituye una respuesta al problema de la indeterminación del derecho. En la segunda parte del ensayo son discutidas, en cambio, las tres teorías de la interpretación que, a partir de la formulación ofrecida por Herbert Hart,

*damiano.canale@unibocconi.it

** Deseo agradecer además a Federico Arena, Francesca Poggi, Giovanni Tuzet y Vito Velluzzi por sus comentarios a una versión anterior de este ensayo. 
polarizan el debate sobre el tema, a saber, la teoría cognitiva, la teoría escéptica y la, así llamada, teoría intermedia ${ }^{1}$. Según Guastini, ninguna de estas teorías provee una descripción adecuada de la praxis interpretativa y por lo tanto ninguna puede, en rigor, presumir del título de "teoría". La interpretación consiste, en realidad, en la atribución a un texto normativo de un significado, elegido entre aquellos que el texto interpretado puede asumir en base a las reglas del lenguaje, a las técnicas interpretativas aceptadas por la comunidad de los intérpretes y a las construcciones conceptuales elaboradas por la dogmática jurídica. Puesto que, en base a estos criterios, cualquier texto normativo puede asumir más de un significado, la elección del intérprete será inevitablemente discrecional y no podrá ser descripta como un acto cognoscitivo. En cambio, los significados que un texto normativo puede asumir son cognoscibles. Esto permite trazar el límite más allá del cual la interpretación, o sea la elección discrecional de uno de los significados posibles, se transforma en creación de normas jurídicas nuevas. Esta reconstrucción, en otro lugar llamada por Guastini "teoría escépticomoderada de la interpretación" ${ }^{2}$, por un lado hace propias algunas tesis fundamentales del escepticismo interpretativo, en particular aquella según la cual la interpretación con fines decisorios comporta siempre una elección discrecional por parte del intérprete; por otro lado permite

${ }^{1}$ H.L.A. Hart, The Concept of Law, 2da. Ed., Oxford, Oxford University Press, 1994, pp. 124 y ss. Para una teoría cognitiva de la interpretación jurídica, esta última consiste en el conocimiento del único significado que un texto normativo posee antes e independientemente de su interpretación; para una teoría escéptica, en cambio, la interpretación consiste en la atribución a un texto normativo de un significado cualquiera, elegido discrecionalmente por el intérprete; para una teoría mixta, por último, en los casos fáciles el juez se limita a conocer el significado que posee el texto jurídico y que regula el caso, mientras en los casos difíciles el juez está obligado a interpretar, o sea a identificar discrecionalmente el significado a atribuir al texto interpretado.

${ }^{2}$ La expresión "teoría escéptico-moderada de la interpretación" se refiere, en realidad, a una familia de teorías que se distinguen entre ellas por el modo en que es caracterizado el marco de los significados admisibles de un enunciado normativo; Véase V. Velluzzi, Interpretazione degli enunciati normativi, linguaggio giuridico, certezza del diritto, en "Criminalia", 2008, p. 494. 
Teorías de la interpretación jurídica...

distinguir entre la interpretación de un texto normativo y la integración del derecho (denominada por Guastini "interpretación creativa", "legislación intersticial" y "construcción jurídica"3), evitando así los resultados contraintuitivos a los que conduce la teoría escéptico-radical.

En esta intervención no discutiré en detalle las tesis apenas mencionadas ni analizaré las distinciones conceptuales sobre las que éstas se basan, presentadas por Guastini con la claridad y la precisión habituales. Concentraré la atención en un tema aparentemente secundario de "El escepticismo ante las reglas replanteado", a saber, la relación entre teorías de la interpretación jurídica y teorías del significado. Según Guastini, una teoría de la interpretación jurídica no necesita una teoría del significado para ser adecuadamente desarrollada ${ }^{4}$. Esto por dos razones:

1. Muy a menudo con la expresión "teoría del significado" -observa Guastini- nos referimos a un discurso prescriptivo, formulado con el objetivo de dirigir ex ante o de criticar ex post lo realizado por los intérpretes jurídicos, en particular los jueces. Un discurso de este tipo no posee, sin embargo, importancia alguna para el teórico de la interpretación jurídica. En efecto, el teórico está preocupado por conocer cómo interpretan de hecho los operadores jurídicos, no en indicar cómo éstos deberían o habrían debido interpretar.

2. Si con "teoría del significado" nos referimos en cambio a un discurso cognoscitivo, tal discurso no podrá más que identificarse con una teoría de la interpretación. Retomando las palabras de Guastini,

una teoría descriptiva de la interpretación es ella misma una teoría descriptiva del significado en contextos jurídicos (y, en

${ }^{3}$ Guastini, "El escepticismo ante las reglas replanteado", cit., p. 31.

${ }^{4}$ Guastini, "El escepticismo ante las reglas replanteado", cit., p. 50. La misma tesis es sostenida por Guastini, en modo más extenso y articulado, en Teoria del significato e teoria dell'interpretazione, ahora en Id., Nuovi studi sull'interpretazione, Roma, Aracne, 2008, pp. 165-174. 
todo caso, la única teoría del significado admisible en contextos jurídicos) ${ }^{5}$.

Si ello es así, se entiende que una teoría de la interpretación no puede presuponer una teoría del significado, puesto que no se trata de dos discursos distintos, de los cuales uno constituye el presupuesto del otro, si no que se trata de un mismo y único discurso.

Esta tesis constituye un corolario de la crítica dirigida, en "E1 escepticismo ante las reglas replanteado", contra la teoría cognitiva de la interpretación recordada en la introducción. Las teorías de la interpretación jurídica de corte cognitivo suelen en efecto ser justificadas, según Guastini, recurriendo a dos "teorías del significado": 1) el significado de un texto jurídico consiste en el contenido literal de este último (teoría del significado literal); 2) el significado de un texto jurídico consiste en la intención de quien lo ha producido (teoría intencionalista). Estas "teorías" no describen, sin embargo, cómo son efectivamente identificados y atribuidos los significados de los textos normativos. Muy a menudo los jueces atribuyen a los textos normativos significados diferentes del literal o del correspondiente a la intención del legislador, suponiendo que éstos existan y sean cognoscibles. Estas presuntas "teorías" han de ser por lo tanto rechazadas, porque no describen la real praxis interpretativa si no que intentan sobre todo influenciarla. Dicho con otras palabras, estas teorías sirven de vehículo a un mal disimulado contenido normativo y, desde un punto de vista teórico, poco importa que ese contenido pueda o no ser compartido sobre la base de las convicciones éticas y políticas de cada uno. A partir de tales consideraciones se seguiría una irrelevancia todavía más general de las teorías del significado para la teoría de la interpretación, desde el momento que las primeras, si no son ya concebidas como discursos directivos, coinciden de hecho con la segunda.

Vale la pena observar cómo la tesis apenas considerada pareciera redimensionar radicalmente la relevancia, para la teoría del derecho, del debate filosófico alrededor de la noción de significado, y que ha

${ }^{5}$ Guastini, "El escepticismo ante las reglas replanteado", cit., p. 50. 
Teorías de la interpretación jurídica...

caracterizado a la filosofía del lenguaje desde Frege hasta nuestros días. En la medida en que este debate no se limita a dar cuenta de las concretas prácticas interpretativas que tienen lugar en una comunidad lingüística, no reviste interés teórico alguno, ni está en grado de captar la especificidad de las prácticas interpretativas en el campo del derecho.

Encuentro sin duda compartible esta última preocupación de Guastini. La interpretación de los textos jurídicos tiene características del todo peculiares, no reducibles a aquellas de la interpretación que tiene lugar en los contextos generalmente considerados por los filósofos del lenguaje, los cuales tienden a privilegiar los usos asertivos o descriptivos del lenguaje natural en contextos conversacionales, como si estos últimos constituyeran un modelo al cual cualquier otro contexto y uso lingüístico puedan ser al fin de cuentas asimilados.

Sin embargo, no comparto la reconstrucción propuesta por Guastini de la relación entre las teorías de la interpretación y las teorías del significado. En realidad, cualquier teoría de la interpretación presupone una teoría del significado, en un sentido de esta expresión que deberemos precisar, aun cuando una teoría del significado jurídico posea características peculiares, que no permiten asimilarla a las teorías del significado de los lenguajes naturales tradicionalmente elaboradas por los filósofos del lenguaje. El lenguaje jurídico, en efecto, tiene la función de guiar los comportamientos humanos y es (en parte) un lenguaje artificial, cuya estructura y contenidos están administrados autoritativamente $^{6}$. Si se prescinde de este hecho, ninguna teoría del significado jurídico que sirva de base a una teoría de la interpretación podrá ser adecuadamente desarrollada.

En esta intervención intentaré en primer lugar evidenciar por qué toda teoría de la interpretación presupone una teoría del significado. Intentaré luego, en segundo lugar, reconstruir brevemente la teoría del significado implícitamente utilizada en "El escepticismo ante las reglas

${ }^{5}$ De "natura amministrata del linguaggio giuridico", si bien en un sentido distinto de aquel propuesto en el texto, habla M. Jori, Definizioni giuridiche e pragmatica, en P. Comanducci-R. Guastini (eds.), Analisi e diritto 1995, Torino, Giappichelli, 1995, p. 129. 


\section{Damiano Canale}

replanteado", evidenciando cómo en realidad el discurso de Guastini presupone dos teorías, entre ellas incompatibles. Este hecho, como veremos, vuelve ambigua la teoría escéptico-moderada, una ambigüedad que es posible poner a la luz analizando la noción de marco de significados admisibles. Si así están las cosas, Guastini debe elegir entre dos posibles especificaciones de su teoría de la interpretación, cada una de las cuales, por un lado, presuponen explicaciones alternativas del funcionamiento del lenguaje y, por otro lado, implican dos modos distintos de concebir el límite entre interpretación e integración del derecho.

\section{Dos tipos de teoría del significado}

Según Guastini, una teoría descriptiva del significado -la única a la que puede, con rigor, darse el nombre de teoría- consiste

simplemente en reconstruir las prácticas interpretativas de una determinada comunidad de intérpretes, por ejemplo, de los hablantes del español (del italiano, del francés, etc.) en sus conversaciones diarias, cuando leen novelas o periódicos, escuchan la radio, etc. ${ }^{7}$.

Debe advertirse que la expresión "reconstruir las prácticas interpretativas" es ambigua. En nuestro contexto de discusión, por "reconstrucción de las prácticas interpretativas" se puede entender:

(a) la identificación y la clasificación de los significados atribuidos (o atribuibles) a un texto normativo;

(b) una explicación de qué es el significado de un texto normativo.

Estos dos tipos de discurso son distintos pero se relacionan entre sí. Son distintos porque la identificación y la clasificación de los significados atribuidos a un texto no explican en qué consiste para el texto en cuestión tener un significado, si no que dan tal explicación por descontada ${ }^{8}$. Están

${ }^{7}$ Guastini, "El escepticismo ante las reglas replanteado", cit., p. 48.

${ }^{8}$ Se podría quizás decir, en la perspectiva de Guastini, que una enumeración de los significados atribuidos es un ejemplo de aquello en lo que consiste para un 
Teorías de la interpretación jurídica....

relacionados porque no es posible identificar y clasificar los significados atribuidos o atribuibles a un texto sin conocer las condiciones de identidad de los significados. Estas condiciones de identidad son necesarias para la identificación del significado y constituyen, tradicionalmente, el objeto de una explicación filosófica de este último.

Los dos tipos de discurso acerca del significado (identificación y clasificación, por un lado, explicación, por el otro) pueden en efecto ser reconducidos a dos contenidos diferentes de la expresión "teoría del significado", contenidos a los que, en el debate filosófico en lengua inglesa, suele hacerse referencia utilizando dos términos distintos, meaning-theory y theory of meaning'.

Una meaning-theory constituye el resultado de una investigación empírica y consiste en la identificación, clasificación y especificación de los significados asumidos por los términos y las expresiones de una cierta lengua objeto, como por ejemplo el inglés o el italiano. Se la puede concebir como una suerte de detallado manual de traducción que registra el comportamiento lingüístico de los miembros de una comunidad de hablantes y obtiene, a partir de éste, los estándares de uso de los términos y de las expresiones de una lengua.

Una theory of meaning constituye, en cambio, el resultado de una investigación de tipo filosófica y consiste en la respuesta a la pregunta siguiente: ¿en qué consiste para una expresión lingüística el tener un significado? Esta pregunta resume, tradicionalmente, al menos tres cuestiones distintas: (1) ¿a qué género de objetos pertenecen los significados? (2) ¿qué tipo de conocimiento es requerido para comprender los significados? (3) ¿en virtud de qué cosa las expresiones de una lengua poseen un determinado significado? La respuesta al problema (1) entra en el campo de estudio de la ontología y se refiere a las condiciones de identidad de los significados; el problema (2) es en

texto el tener significado. Se trata de un ejemplo que, de todos modos, presupone lo que se propone ejemplificar, o sea las condiciones de identidad de los significados lingüísticos.

${ }^{9}$ La distinción entre meaning-theories y theories of meaning se debe a M. Dummett, What Is a Theory of Meaning? (I), ahora en Id., The Seas of Language, Oxford, Clarendon Press, 1996, pp. 1-22. 
cambio de tipo epistemológico y está hoy en el centro de numerosos debates filosóficos, tanto acerca de la relación entre semántica y pragmática como acerca de la naturaleza de la competencia lingüística; el problema (3), finalmente, reenvía a consideraciones que podríamos llamar, genéricamente, causales y está en la base de los programas actuales que persiguen la naturalización de la filosofía del lenguaje.

Ahora bien, cuando Guastini usa la expresión "teoría del significado" y afirma que, entendida como discurso descriptivo, coincide con una teoría de la interpretación, parece a primera vista referirse a una meaningtheory, o sea a la identificación y clasificación de las reglas de uso de los términos y de las expresiones pertenecientes al lenguaje de las fuentes.

Sin embargo, en base a lo observado hasta aquí, toda meaning-theory presupone una theory of meaning. En efecto, un lenguaje natural contiene, potencialmente, un número infinito de términos y de expresiones lingüísticas. Para especificar el significado de cada término y expresión no es suficiente, por lo tanto, desarrollar una investigación empírica, que tenga por objeto regularidades lingüísticas observables, si no que es necesario fijar, como una hipótesis de trabajo, las condiciones de identidad de los significados, investigando además qué tipo de comportamiento, habilidad o conocimiento implica el tratar a una expresión como dotada de significado y/o cuáles circunstancias concurren a hacer que tal expresión adquiera un cierto significado. Solo bajo estas condiciones la investigación empírica podrá producir resultados determinados y dar respuestas interesantes a los problemas que estamos considerando. En breve, para construir una meaning-theory el estudioso debe hacer uso -en modo explícito o implícito, consciente o inconscientemente- de una theory of meaning que dé una explicación general del funcionamiento del lenguaje.

Es necesario preguntarse, por lo tanto, por qué razón Guastini no considera este aspecto de la cuestión y, más aún, niega la relevancia de una theory of meaning para la elaboración de una genuina teoría de la interpretación jurídica. Me parece que este problema puede encontrar dos respuestas diferentes e igualmente plausibles dentro del discurso de Guastini.

La primera respuesta podría ser la siguiente, las theories of meaning no son discursos filosóficos rigurosamente analíticos y descriptivos; su 
objeto, además, no está claramente definido. Conviene por lo tanto desconfiar de este tipo de discursos, ya que existe el riesgo de que escondan posiciones de tipo ideológico y, de todos modos, no son necesarios para la construcción de una teoría de la interpretación. Retomaré esta tesis en la última sección de este ensayo; quisiera aquí en cambio concentrarme en una respuesta alternativa al problema, ciertamente más interesante que la primera. Si dejamos de lado una genérica desconfianza con respecto a las theories of meaning usualmente desarrolladas por los filósofos, la razón de la posición de Guastini podría ser, en cambio, de tipo exquisitamente filosófica. Vista de cerca la tesis de la irrelevancia de una teoría del significado, para el desarrollo de una teoría de la interpretación, presupone, en efecto, una explicación general del funcionamiento del lenguaje. Se podría entonces decir que, al afirmar la irrelevancia de una theory of meaning para el estudio teórico de la interpretación, Guastini de hecho adhiere, implícitamente, a una theory of meaning ${ }^{10}$.

¿Cuáles son sus contenidos?

${ }^{10}$ Según Pierluigi Chiassoni la tesis en base a la cual "a los fines de una teoría de la interpretación jurídica (...) no es necesario recurrir a alguna teoría del significado", no puede ser entendida literalmente. Se trata sobre todo de una tesis epistemológica, según la cual el conocimiento jurídico es asimilable a una forma de "sociología descriptiva" que incluye "un trabajo de reconstrucción conceptual de los fenómenos interpretativos, con la finalidad de elaborar un modelo representativo claro" (P. Chiassoni, L'ineluttabile scetticismo della "scuola genovese", en P. Comanducci-R. Guastini (ed.), Analisi e diritto 1998, Torino, Giappichelli, 1998, pp. 47-48). Mi tesis en esta intervención es que el trabajo de reconstrucción conceptual, necesario para elaborar un modelo claro de los fenómenos interpretativos, puede ser llevado a cabo solamente con el auxilio de una theory of meaning, en el sentido especificado en el texto. Sobre este punto veáse también M. Barberis, Lo scetticismo immaginario. Nove obiezioni agli scettici à la génoise, en P. Comanducci-R. Guastini (eds.), Analisi e diritto 2000, Torino, Giappichelli 2000, pp. 15 y ss. Sobre la relación entre teorías del significado y teoría de la interpretación véase también V. Villa, Interpretazione giuridica e teorie del significato, en L. Gianformaggio-M. Jori (eds.), Scritti per Uberto Scarpelli, Milano, Giuffrè, 1997, pp. 803-853.

Ha de notarse que la tesis aquí propuesta es en realidad compatible con aquella defendida por Guastini. El enunciado "a los fines de una teoría de la 


\section{Una comparación entre dos familias de teorías}

Son innumerables las explicaciones filosóficas que, en el debate contemporáneo, compiten por explicar el funcionamiento de los lenguajes naturales. Se trata de una "competición" de la cual no es fácil dar un resumen, porque cada theory of meaning se introduce dentro de programas de investigación distintos, que focalizan la atención sobre aspectos diferentes del lenguaje y desarrollan con este objeto instrumentos conceptuales no siempre comparables entre sí.

A nuestros fines, sin embargo, es suficiente notar, en términos generalísimos, cómo las diferentes theories of meaning pueden ser clasificadas en dos grandes familias, las teorias proposicionales y las teorias interpretativas, que representan dos enfoques filosóficos distintos del problema del significado. Consideremos estas familias con mayor detalle $\mathrm{e}^{11}$.

interpretación jurídica (...) no es necesario recurrir a alguna teoría del significado" es en efecto ambiguo. Este enunciado puede significar "ninguna de las teorías del significado actualmente disponibles en la literatura resulta necesaria para construir una teoría de la interpretación jurídica", o bien "para construir una teoría de la interpretación jurídica no es necesario recurrir a una teoría del significado, en cualquier modo que sea ésta concebida". En la primera acepción de sentido, la expresión "teoría del significado" recurre como token; en la segunda acepción de sentido, en cambio, la misma expresión recurre en cambio como type. A la luz de la reconstrucción aquí propuesta, por lo tanto, la tesis de Guastini es errónea si la expresión "teoría del significado" es usada como type; la tesis es en cambio correcta si la misma expresión es usada como token.

${ }^{11}$ Se podría objetar que falta aquí mencionar una tercera familia de teorías del significado, no menos importante respecto a aquellas apenas mencionadas, a saber, la familia de las teorías del significado como uso inspiradas en la filosofía de Ludwig Wittgenstein. Ahora bien, aun cuando la idea de que los significados dependan de los usos lingüísticos se encuentre hoy ampliamente difundida también entre los juristas, el concebir el significado de una palabra "como su uso en el lenguaje" (L. Wittgenstein, Ricerche filosofiche, Torino, Einaudi, 1995, § 43) tiene consecuencias relevantes sobre el modo mismo de abordar el estudio del lenguaje, que excluyen la posibilidad de que una teoría del significado, concebida como sea, pueda llevar a cabo la función cognitiva 
Teorías de la interpretación jurídica...:

\subsection{Teorías proposicionales del significado}

Según este primer enfoque filosófico, los significados son entidades complejas que permiten que las expresiones de un lenguaje entren en relación con objetos, estados de cosas, eventos en el mundo. Tales entidades son concebidas en modos muy diferentes por las theories of meaning que pertenecen a esta familia. A título puramente ejemplificativo, los significados son a veces descriptos como objetos realmente existentes situados en un mundo distinto del físico ${ }^{12}$; como meras estipulaciones internas de una teoría que proporcionan los recursos lingüísticos suficientes para describir los fenómenos observados con ciertos fines prácticos ${ }^{13}$; como convenciones que guían los usos del lenguaje dentro de una cierta comunidad ${ }^{14}$; incluso, como eventos cognitivos o intencionales que tienen lugar cuando los hablantes predican propiedades o relaciones entre entes o entre estados de $\operatorname{cosas}^{15}$.

que le viene comúnmente atribuida. Si se acoge una concepción del significado como uso, en efecto, desaparece la posibilidad de dar una explicación general del funcionamiento del lenguaje, algo que en cambio pretende lograr una theory of meaning. El estudioso deberá conformarse con explicaciones locales, focalizadas en algunas semejanzas de familia entre usos lingüísticos particulares. Una concepción del significado como uso, además, excluye la posibilidad de que la comprensión del significado de una expresión lingüística permita dominar sus aplicaciones futuras, volviendo por lo tanto falta de utilidad e interés cualquier meaning-theory. Estando así las cosas, no se puede considerar esta posición filosófica como una teoría del significado junto a otras, dado que ésta lanza un ataque radical a la idea misma de teoría. Esto explica por qué cuando los teóricos del derecho y los juristas afirman que el significado depende del uso, en realidad están pensando en una teoría proposicional o una teoría interpretativa del significado, pace Wittgestein.

${ }^{12}$ G. Frege, Der Gedanke. Eine logische Unitesuchung, "Beiträge zur Philosophie des deutschen Idealismus", 1, 1918, pp. 58-77.

${ }^{13}$ R. Carnap, Meaning and Necessity, Chicago-London, The University of Chicago Press, 1956, pp. 205 y ss.

${ }^{14}$ D. Lewis, Convention. A Philosophical Study, Oxford, Oxford University Press, 2002, pp. 160 y ss.

${ }^{15}$ S. Soames, What Is Meaning?, Princeton, Princeton University Press, 2010. 
Según esta perspectiva filosófica, los significados son por lo tanto anteriores a cada una de las instancias particulares de uso del lenguaje y el conocimiento que implica tratar a una expresión como dotada de significado es de tipo proposicional. Estas consideraciones pueden ser extendidas también a los enunciados deónticos, los cuales se prestan a ser descriptos como compuestos de una parte referencial, constituida de una proposición, a la cual se agrega una parte no referencial (expresiones como "es obligatorio que", "se debe", etc.), que explicita el acto lingüístico cumplido al pronunciar el enunciado deóntico completo $^{16}$. Esta familia de teorías, además, no reconoce a los significados propiedades causales; no obstante, se considera que los significados dependen de los contextos de comunicación lingüística, los cuales contribuyen a su determinación y a su completamiento cuando sea necesario. Por cuanto concierne, finalmente, a la relación entre significado e interpretación, este enfoque filosófico no permite concebir a los significados como el producto de una actividad interpretativa. Si bien el término "interpretación" puede designar tres actividades distintas, cada una de ellas presupone la existencia de los significados. Así, interpretar puede querer decir, o bien conocer los significados, o bien reducir su indeterminación, o bien seleccionar los significados adecuados para conseguir determinados fines prácticos. Por lo tanto, una teoría del significado no coincide con una teoría de la interpretación, sino que esta última presupone la primera.

Es oportuno en este punto preguntarse, ¿este enfoque filosófico es idóneo para dar cuenta de los significados jurídicos? Dicho de otro modo, ¿una teoría de los significados jurídicos puede asumir la forma de una teoría proposicional del significado?

Bajo ciertos aspectos parecería que sí, la explicación del funcionamiento del lenguaje ofrecida por este enfoque es coherente con algunas intuiciones difundidas entre los juristas y los teóricos del

\footnotetext{
${ }^{16}$ R.M. Hare, The Language of Morals, Oxford, Oxford University Press, 1991; C.E. Alchourrón-E. Bulygin, The Expressive Conception of Norms, in R. Hilpinen (ed.), New Studies in Deontic Logic: Norms, Actions and the Foundation of Ethics, Springer, Dordrecht, 1981, pp. 95-124.
} 
Teorías de la interpretación jurídica...

derecho. Comprendemos el significado de un texto normativo sobre la base de su estructura sintáctica y de las propiedades semánticas de las palabras que lo componen. Tales propiedades son reconocidas en términos y expresiones por una comunidad lingüística y constituyen el resultado de convenciones, estipulaciones, eventos cognitivos o intencionales susceptibles de ser analizados y descriptos. Tales convenciones, estipulaciones o eventos, a su vez, individualizan los significados estándar de una expresión lingüística, o sea los significados que tal expresión asume con mayor frecuencia, o sea (en el caso que se reconozcan propiedades normativas a los significados) los significados correspondientes a las reglas de uso del lenguaje ${ }^{17}$. Se trata de significados públicos, compartidos por los hablantes en cada una de las instancias de uso de los términos y expresiones; significados que hacen posible y guían la comunicación lingüística. Esto no implica que un término o una expresión lingüística pueda asumir un único significado, y por lo tanto que un enunciado interpretativo de forma "T significa S", usado por el juez en modo adscriptivo, pueda ser considerado verdadero o falso. Una teoría proposicional del significado sirve incluso para explicar por qué una misma expresión lingüística asume de hecho más significados, como también por qué un mismo significado puede ser expresado por expresiones lingüísticas diferentes. La noción de significado estándar individua simplemente la clase de significados que pueden ser expresados mediante un texto o una proferencia.

Por otro lado, sin embargo, una teoría proposicional del significado oscurece algunas características peculiares del lenguaje jurídico. En primer lugar, pone en segundo plano la función adscriptiva que, en sede decisoria, llevan a cabo los enunciados interpretativos; privilegiando el papel desempeñado, en la comunicación lingüística, por el contenido proposicional de tales enunciados y el carácter público de este último. Bajo el perfil lógico, sin embargo, los enunciados interpretativos pueden llevar a cabo una función adscriptiva más allá de los significados estándares. En este sentido, el proferir "T significa S", por parte de un

${ }^{17}$ Sobre la noción de significado estándar véase $\mathrm{D}$. Marconi, $A$ che cosa serve il significato, "Rivista di filosofia", 102 (1), 2011, pp. 29-46. 


\section{Damiano Canale}

juez en sede decisoria, constituye un acto "feliz" de atribución de significado. Un acto que desempeña la propia función comunicativa, más allá del hecho que $\mathrm{S}$ entre en la categoría de los significados estándar de T. Incluso más, la atribución de significado es capaz de generar estándares semánticos. Esto conduce a dudar de la necesidad de recurrir a las nociones de "lenguaje público" y "significados compartidos" para explicar por qué un texto normativo posee un determinado significado. La existencia de significados compartidos parece, en efecto, no ser una condición necesaria o suficiente para el funcionamiento del lenguaje en el contexto jurídico. Eso no impide, obviamente, que los textos normativos sean de hecho interpretados en modo similar por los operadores jurídicos. Banalmente, vale la pena recordar que del hecho de que los intérpretes se comporten de manera similar no está justificado inferir que existan significados compartidos; tanto como el hecho de que los sistemas cardiovasculares humanos sean similares entre sí no nos autoriza a hablar de un sistema cardiocirculatorio público ${ }^{18}$.

\subsection{Teorías interpretativas del significado}

Según un enfoque interpretativo del problema del significado para explicar el funcionamiento del lenguaje no es necesario concebir ningún misterioso intermediario -los significados, las proposiciones, las normas u otra cosa- entre las expresiones lingüísticas y aquello con lo que estas últimas se relacionan ${ }^{19}$. Las nociones familiares de verdad, referencia, eficacia y satisfacción son más que suficientes para desempeñar este papel explicativo. Dado un enunciado interpretativo "T significa S", utilizado en función cognitiva, podemos, por ejemplo, construir un enunciado equivalente de la forma "T es verdadero si y solo si S", donde $\mathrm{S}$ constituye la interpretación o traducción de $\mathrm{T}$ a un determinado

\footnotetext{
${ }^{18}$ Véase N. Chomsky, Explaining Language Use, en Id., New Horizons in the Study of Language and Mind, Cambridge, Cambridge University Press, 2000, pp. 19 y ss.

${ }^{19}$ Véase W.V.O. Quine, Philosophy of Logic, 2da ed., Cambridge (MA), Harvard University Press, 1986, cap. 1.
} 
lenguaje objeto. Si S es una interpretación o traducción adecuada, capaz de satisfacer las condiciones de verdad de $\mathrm{T}$, entonces este bicondicional será capaz de desempeñar todas las funciones explicativas que normalmente se exigen a una teoría del significado. Así, la noción de significado cognoscitivo puede ser eficazmente reemplazada por la noción de condición de verdad, y por lo tanto las tareas de la theory of meaning podrán ser adecuadamente llevadas a cabo por una theory of interpretation. Este tipo de análisis, visto de cerca, puede ser extendido a los enunciados interpretativos utilizados en función adscriptiva en el caso que se les reconozca el valor semántico de la eficacia, parasitario respecto al de verdad. En este caso, el enunciado "T significa S" es equivalente a "T es eficaz si y solo si S", donde S expresa la obediencia, la observancia, el cumplimiento o la satisfacción de $\mathrm{T}$ por parte de sus destinatarios $^{20}$. Se sigue que la noción de significado normativo puede ser felizmente sustituida por la noción de condición de eficacia, y que la función explicativa tradicionalmente atribuida a una theory of meaning puede ser llevada a cabo, también en el análisis del lenguaje normativo, por una theory of interpretation.

Es importante subrayar cómo, ya sea en el caso de los enunciados cognoscitivos o en el caso de los enunciados normativos, la adecuada interpretación o traducción $\mathrm{S}$ de $\mathrm{T}$ constituye una cuestión de hecho, que depende de los comportamientos y de las actitudes observables de quien usa el lenguaje $\mathrm{e}^{21}$. Una teoría de la interpretación, para desempeñar el papel que se le atribuye, deberá por lo tanto comenzar por la observación empírica de la interacción lingüística entre los hablantes y extraer de ésta un modelo capaz de explicar por qué la emisión de una determinada proferencia provoca una determinada reacción en su destinatario, qué tipo de actividad y de conocimiento están implicadas

20 Véase J.J. Moreso, La indeterminación del derecho y la interpretación de la Constitución, Madrid, Centro de Estudios Políticos y Constitucionales, 1997, pp. 24 y ss. Véase también B. Celano, Efficacia, anti-realismo, interpretazione, en P. Comanducci-R. Guastini (eds.), Analisi e diritto 1999, Torino, Giappichelli, 1999, pp. 229-247.

${ }^{21}$ D. Davidson, Radical Interpretation, en Id., Inquires into Truth and Interpretation, Oxford, Oxford University Press, 1984, pp. 125-140. 
en este hecho, y, en definitiva, por qué la comunicación lingüística funciona.

Los modelos explicativos desarrollados por los filósofos del lenguaje con este objetivo son bastante diferentes. El éxito de la comunicación puede ser explicado sobre la base de una serie de hipótesis, formuladas implícitamente por los hablantes, en mérito a cómo el interlocutor usará el lenguaje (teoría anterior), y por ulteriores hipótesis acerca del modo en que el interlocutor está de hecho usando el lenguaje (teoría transitoria), en modo tal de adaptar continuamente las hipótesis de partida del hablante al desarrollo del intercambio lingüístico ${ }^{22}$. El mismo fenómeno puede ser explicado también en términos "contractuales", la interacción entre los hablantes se basa sobre estipulaciones y acuerdos implícitos, alcanzados en cada intercambio lingüístico o serie de intercambios lingüísticos. Acuerdos mediante los cuales se introducen nuevos términos, se asignan nuevos significados a términos ya en uso o se utilizan significados concordados con anterioridad, según las circunstancias $^{23}$. Una teoría interpretativa del significado es además capaz de dar cuenta de las variables contextuales que condicionan la comunicación y de los diferentes tipos de actos lingüísticos cumplidos al pronunciar un enunciado, gracias al soporte ofrecido por la lógica modal y por la noción de mundo posible. En efecto, el "significado" de un enunciado puede ser descripto como una función, de mundos posibles a valores de verdad ${ }^{24}$, en la cual el contexto se corresponde con las características relevantes del mundo en el cual el enunciado es verdadero o eficaz, mientras el tipo de acto lingüístico realizado por los hablantes se corresponde con una relación de ajuste entre mundos.

Vale la pena notar que según este enfoque filosófico los "significados" no son el presupuesto de la interpretación, por el contrario, lo que

22 D. Davidson, A Nice Derangement of Epitaphs, en Id., Truth, Language and History, Oxford, Clarendon Press, 2005, pp. 89-125. La competencia semántica de los hablantes consiste, en este caso, en su capacidad de elaborar teorías transitorias y eficientes.

${ }^{23}$ P. Lidlow, Cheap Contextualism, "Philosophical Issues", 18, 2008, pp. 104-129.

24 J. Barwise-J. Perry, Situations and Attitudes, Cambridge (MA), MIT Press, 1983. 
Teorías de la interpretación jurídica...

comúnmente llamamos "significado" constituye el resultado de un acto interpretativo. El conocimiento primario implicado en la comprensión del lenguaje no es por lo tanto de tipo proposicional (knowing-that) sino de tipo práctico (knowing-how), consiste en la habilidad para responder adecuadamente a los estímulos lingüísticos provenientes de los demás, volviéndolos así significantes; una habilidad práctica de la cual a su vez depende el conocimiento de tipo proposicional. Al mismo tiempo, si bien los significados, como tales, no existen y no poseen por lo tanto propiedades, la función explicativa que tradicionalmente se les atribuye puede ser llevada a cabo por relaciones de tipo causal, dado que éstas últimas se demuestran capaces de explicar la interacción lingüística. Debe advertirse, además, que este enfoque no considera la existencia de convenciones lingüísticas y de un lenguaje público como una condición necesaria o suficiente de funcionamiento del lenguaje. Es decir, existen solamente situaciones comunicativas singulares o locales, a menudo relacionadas entre sí, que pueden ser descriptas en términos pragmáticos y que pueden ser explicadas por una teoría de la interpretación adecuadamente formulada, sin que sea necesario traer a colación las nociones de lenguaje público y de significados compartidos.

También en este caso vale la pena preguntarse, ¿el enfoque filosófico apenas esbozado es capaz de dar cuenta del funcionamiento del lenguaje jurídico? A primera vista la respuesta parece ser positiva. Si un análisis veritativo-funcional puede ser extendido a los enunciados normativos -no con referencia a su presunto contenido proposicional si no a su eficacia, la cual, a diferencia de las proposiciones, es empíricamente observable-, entonces una teoría interpretativa del significado da claramente cuenta del funcionamiento del lenguaje jurídico, y permite poner en primer plano las diferentes funciones (cognitiva, propositiva, adscriptiva, etc.) que los enunciados interpretativos desempeñan en cada uno de los diferentes contextos en que son usados ${ }^{25}$. Sin embargo, asumir este punto de vista hace más problemático explicar la regularidad

${ }^{25}$ Para una tipología de los enunciados interpretativos basada en la función enunciativa que desempeñan véase Chiassoni, L'ineluttabile scetticismo della "scuola genovese", cit., pp. 23 y ss. 
de los comportamientos lingüísticos de los intérpretes, o sea, dar cuenta del hecho que los intérpretes tienden a adecuarse a los significados estándares que, bajo esta perspectiva, son meramente accidentales y contingentes ${ }^{26}$.

\subsection{Algunas observaciones al margen}

Las características de estas dos familias de thoeries of meaning merecen algunas observaciones con referencia al tema de nuestra investigación:

(a) Ni de un enfoque proposicional ni de un enfoque interpretativo de la teoría del significado se sigue que exista una relación biunívoca entre un texto y una de sus interpretaciones. Es decir, a un texto $\mathrm{T}$ no corresponde necesariamente una y solo una interpretación I, así como a una interpretación I no corresponde necesariamente uno y solo un texto T. Al negar la relevancia de una teoría del significado para la teoría de la interpretación, Guastini parece al contrario movido por una preocupación de signo opuesto, a saber, inevitablemente el recurso a una teoría del significado volvería la noción de significado independiente de la noción de interpretación, al punto de poner en discusión algunos axiomas de la teoría escéptico-moderada, tales como la distinción entre disposición y norma, y el hecho de que cualquier disposición expresa potencialmente numerosas normas. En base a cuanto hasta aquí hemos observado, esta preocupación no se encuentra sin embargo justificada. Tanto las teorías proposicionales como las teorías interpretativas del significado no ponen en duda la pluralidad semántica y pragmática de los enunciados lingüísticos, ni el carácter discrecional de las elecciones interpretativas.

${ }^{26}$ Para una discusión profunda sobre este problema véase D. Marconi, La competenza lessicale, Roma-Bari, Laterza, 1999. Según numerosos autores las semejanzas y las regularidades de uso del lenguaje son más que suficientes para justificar el recurso a la noción de lenguaje público y significados compartidos. Véase M. Devitt, Ignorance of Language, Oxford, Oxford Univesity Press, 2006, pp. 163 y ss. 
(b) Los discursos que Guastini llama "teoría del significado", o sea la teoría del significado literal y la teoría intencionalista recordadas anteriormente, no presentan ni las características de una meaningtheory ni aquellas de una theory of meaning. En efecto, estos discursos no proveen, ni una clasificación de las interpretaciones efectuadas por los juristas, ni una explicación del funcionamiento del lenguaje. Los discursos a los cuales se refiere Guastini formulan simplemente algunas directivas interpretativas destinadas a orientar y justificar la interpretación. Hace bien por lo tanto Guastini en subrayar el carácter prescriptivo de estos discursos. No resulta en cambio justificada la etiqueta de "teorías del significado" que les atribuye, como tampoco resulta justificada la tesis en base a la cual, cualquier teoría del significado que no coincidiese con una teoría de la interpretación, tendría un carácter prescriptivo. Las familias de teorías antes mencionadas están compuestas de discursos analíticos y descriptivos que se proponen explicar hechos sociales, y no guiar o justificar el comportamiento de los intérpretes.

(c) Una Theory of meaning puede pertenecer a la familia de las teorías proposicionales o bien a la familia de las teorías interpretativas, pero no puede pertenecer a ambas. La explicación del funcionamiento del lenguaje ofrecida por el primer enfoque es en efecto incompatible con aquella ofrecida por el segundo. Bastan algunos ejemplos para evidenciar esta circunstancia. Así, las teorías proposicionales afirman la existencia de los significados, mientras que las interpretativas consideran que los significados no existen como entidades independientes de la interpretación; las teorías proposicionales consideran que una teoría de la interpretación es parasitaria respecto a una teoría del significado, mientras las teorías interpretativas afirman la tesis opuesta, e incluso consideran que la teoría de la interpretación puede tranquilamente desempeñar la propia función cognitiva sin recurrir a la noción de significado; las teorías proposicionales consideran que la comunicación lingüística tiene lugar porque existe un lenguaje público, mientras las teorías interpretativas niegan que tal lenguaje exista y explican cómo funciona la comunicación lingüística sin apelar a la noción de lenguaje público. 
La construcción de una teoría de la interpretación coherente necesita por lo tanto de una elección de campo entre estas dos perspectivas filosóficas. Como alternativa, el teórico de la interpretación tiene la obligación de encontrar una mediación coherente entre éstas o bien de elaborar una teoría del significado original, no atribuible a estos dos enfoques ${ }^{27}$.

(d) Los dos enfoques filosóficos examinados no logran, en la formulación estándar ofrecida por los filósofos del lenguaje, explicar en modo exhaustivo el funcionamiento del lenguaje jurídico. Ambas perspectivas concentran la atención sobre los usos asertivos del lenguaje, que tiene lugar en la conversación entre dos hablantes. Pero en los contextos jurídicos lo que cuenta es, sobre todo, el uso normativo de textos autoritativos en la comunicación que tiene lugar en ámbito institucional. Estos enfoques deben por lo tanto ser enmendados y/o integrados por el filósofo del derecho, no simplemente adoptados como modelos listos para usar. En otros

27 Asumiendo este desafío, en algunos de mis escritos, he elaborado una teoría del significado jurídico que intenta superar la dicotomía entre enfoque proposicional y enfoque interpretativo, una teoría deudora del trabajo filosófico de Donald Davidson, David Lewis y Robert Brandom. En base a esta teoría, que podríamos denominar "teoría inferencial del significado jurídico", el significado de una disposición normativa está constituido por el conjunto de inferencias en las que los términos y las expresiones que la componen se ven involucradas dentro del razonamiento de los juristas. Tales inferencias se prestan a ser descriptas como hechos que instancian la observancia de las reglas de uso del lenguaje jurídico en un determinado contexto, haciendo explícita la competencia sintáctica, semántica y pragmática de los hablantes. Estas reglas de inferencia, por un lado, forman parte del bagaje de conocimiento que el jurista adquiere para llevar a cabo su propio trabajo, por otro lado, son objeto de continuas "tratativas", sobre todo en sede decisoria. El modo en el que estas tratativas se producen puede ser descripto mediante el modelo del "contador de puntaje" (scorekeeping) en un juego de interacción estratégica, un modelo ideado por David Lewis y perfeccionado por Robert Brandom. Véase D. Canale, Forme del limite nell'interpretazione giudiziale, Padova, CEDAM, 2003; Id., Inferenzialismo semantico e ragionamento giuridico, "Ragion pratica", 25, 2005, pp. 301-334. 
Teorías de la interpretación jurídica....

términos, para dar cuenta del funcionamiento del lenguaje jurídico, el filósofo del derecho no puede contentarse con utilizar los instrumentos conceptuales estándares elaborados por la filosofía del lenguaje; al contrario, el estudio del lenguaje jurídico, en virtud de la peculiaridad de su objeto, puede dar una contribución relevante al debate filosófico general, evidenciando sus límites y proponiendo líneas de investigación originales.

\section{4. ¿Una o dos teorías de la interpretación?}

Lo dicho en la sección precedente, más allá de su generalidad, es suficiente para poner en evidencia los presupuestos implícitos de la teoría escéptico-moderada de la interpretación. El problema que podríamos en este punto plantearnos es, en efecto, el siguiente: si toda teoría de la interpretación presupone una theory of meaning, ¿cuál es la theory of meaning presupuesta por la teoría de la interpretación formulada por Guastini?

La respuesta a este problema es doble, en el sentido que Guastini adhiere implícitamente a dos distintas theories of meaning, entre ellas incompatibles.

Para evidenciar esto, consideremos en primer lugar la interpretación decisoria, o sea la interpretación efectuada en sede decisoria por los jueces y por los funcionarios administrativos. Al describir sus características, Guastini parece hacer propios, implícitamente, los presupuestos fundamentales de una teoría interpretativa del significado. Numerosos son los indicios que apoyan esta hipótesis. Guastini no deja de remarcar cómo la interpretación consiste en la atribución de un significado a un texto normativo, una actividad considerada similar a la traducción y cuyos resultados son asimilables a las definiciones estipulativas ${ }^{28}$, las cuales, a diferencia de las definiciones lexicales, no se limitan a enumerar los significados que un término puede asumir sino que hacen

${ }^{28}$ Véase R. Guastini, Interpretive Statement, en E. Garzón Valdéz, W. Krawietz, G.H. von Wright, R. Zimmerling (eds.), Normative Systems in Legal and Moral Theory. Festschrift for Carlos E. Alchourron und Eugenio Bulygin, Berlin, Duncker \& Humblot, 1997, pp. 279-298. 
que un término signifique algo que antes no significaba. Además, tanto la definición de interpretación cuanto la definición de enunciado interpretativo que encontramos en los trabajos de Guastini no necesitan, vistas de cerca, recurrir a la noción de significado. La expresión "atribuir el significado S a T" es en efecto equivalente a "reformular T como R", donde $\mathrm{T}$ es el enunciado interpretado, $\mathrm{R}$ es el enunciado interpretante, y el término "significado" no aparece. Esto pone en evidencia cómo Guastini, mediante el término "significado", no se refiere a una misteriosa entidad, distinta de los enunciados y que constituiría el contenido proposicional de estos últimos, sino a una relación entre un enunciado interpretado y un enunciado interpretante, instituida por un específico acto lingüístico. Las mismas consideraciones se pueden extender a la noción de enunciado interpretativo, cuya forma " $T$ significa S" puede ser más claramente presentada como " $\mathrm{R}$ reformula $\mathrm{T}$ en C", donde C indica el contexto de tipo cognoscitivo, propositivo o decisorio en el cual la interpretación tiene lugar.

Estas observaciones ponen en evidencia que para Guastini no es necesario recurrir a la noción de significado para describir en modo claro en qué consiste la interpretación. Vale incluso lo contrario, para explicar qué se entiende comúnmente por significado -si bien los significados, como entidades distintas de los enunciados, no existen- es necesario recurrir a la noción de interpretación. Eso hace que no sea necesario hipostasiar la existencia de un lenguaje público, cuyos contenidos serían compartidos por los miembros de una comunidad lingüística, para explicar en qué consiste la interpretación, la cual se caracteriza, sobre todo, por el tipo de acción cumplida por el intérprete cuando reformula un texto normativo.

Concentrémonos ahora, en segundo lugar, en la interpretación cognitiva, o sea la interpretación realizada por el estudioso del derecho para identificar los significados que un texto normativo puede asumir, en base a las reglas de la lengua, los estándares interpretativos y las distinciones dogmáticas de una cierta comunidad jurídica. Al describir las características de esta species del genus interpretación, Guastini adhiere en cambio, si bien implícitamente, a una teoría proposicional del significado. También en este caso los indicios que sostienen esta 
Teorías de la interpretación jurídica...

hipótesis son numerosos. Al inicio de "El escepticismo ante las reglas replanteado", así como en muchos otros escritos, Guastini hace uso de la distinción entre sentido y referencia introducida por Frege, que constituye uno de los puntos cardinales de las teorías proposicionales sucesivas. Aun cuando el significado de un enunciado sea expresable mediante otro enunciado, la interpretación es de todos modos descripta como el proceso que conduce de los enunciados a los significados, desde el momento que "interpretar es identificar significados, no textos -y no es posible aclarar decentemente en qué consiste la interpretación sin esta distinción"29. Dicho de otro modo, para explicar en qué consiste la interpretación y dar una teoría, es indispensable utilizar la noción de significado, aun cuando se trate de una estipulación interna a la misma teoría ${ }^{30}$. La noción de interpretación cognitiva, además, presupone que los significados sean públicos, conocidos por todos los miembros de una comunidad lingüística y que existan ya antes de ser atribuidos a un término o expresión de un lenguaje ${ }^{31}$. En efecto, solo en el caso que tales significados existan y sean cognoscibles antes que la interpretación decisoria tenga lugar el juez podrá elegir uno de ellos con fines decisorios. Así mismo, en base a un enfoque proposicional, si no existiese un lenguaje público y significados compartidos no podríamos distinguir entre la interpretación de los textos normativos y la integración del derecho, uno de los puntos cardinales de la teoría escéptico-moderada.

Claramente, a la luz de estas observaciones, las características de la interpretación cognitiva presuponen una concepción del funcionamiento del lenguaje incompatible con aquella que Guastini parece adoptar,

${ }^{29}$ R. Guastini, Una teoria cognitiva dell'interpretazione, en Id., Nuovi studi sull'interpretazione, cit., p. 91.

${ }^{30}$ Este modo de concebir los significados, de clara derivación carnappiana, surge con claridad en Guastini, Una teoria cognitiva dell'interpretazione, cit., p. 90.

${ }^{31}$ Comentando la tesis fundamental de la teoría escéptica de la interpretación, en base a la cual "no existe ningún significado antes de la interpretación", Guastini observa, en efecto, que "los filósofos del derecho no han todavía ofrecido otro argumento que permita defender con seriedad esa tesis" (Guastini, "El escepticismo ante las reglas replanteado", cit., p. 51). 


\section{Damiano Canale}

implícitamente, cuando describe las características de la interpretación decisoria. Con esto no entiendo sostener, nótese bien, que en el discurso de Guastini encuentren formulación dos teorías distintas de la interpretación, una de tipo cognitiva, con referencia a la interpretación cognitiva, y la otra de tipo escéptico, con referencia a la interpretación decisoria. Circunstancia, ésta, que volvería el discurso autocontradictorio. Una objeción de este tipo es evitada por Guastini precisando que lo que distingue a la interpretación decisoria de aquella cognitiva es simplemente el tipo de acto lingüístico cumplido por el intérprete. Utilizando el léxico de Austin, podríamos decir que en el caso de la interpretación cognitiva el intérprete cumple un acto de constatación, el intérprete describe el hecho que el significado $\mathrm{S}$ es atribuible a T. En el caso de la interpretación decisoria, el intérprete cumple en cambio un acto performativo de tipo adscriptivo, atribuyendo a $\mathrm{T}$ el significado $\mathrm{S}^{32}$.

La tesis que quisiera sostener en esta sede es otra. Me parece que, cuando construye la teoría escéptico-moderada de la interpretación, Guastini hace referencia implícitamente a dos theories of meaning incompatibles entre ellas. Esto vuelve su teoría, no autocontradictoria pero sí, ambigua, si bien tal ambigüedad pueda ser fuente de contradicciones en el caso que sean explicitados los presupuestos y las consecuencias de la teoría misma.

\section{Marco a priori y marco a posteriori}

La ambigüedad que he apenas mencionado puede ser advertida si consideramos más atentamente la noción de marco o límite de los significados admisibles, que la interpretación cognitiva permitiría trazar. Vista de cerca, la noción de marco puede asumir dos significados distintos, dependiendo de la theory of meaning implícitamente utilizada por quien usa la noción en sede teórica.

${ }^{32}$ Véase Guastini, Teoria del significato e teoria dell'interpretazione, cit., p. 168, nota 11. 
Si adoptamos una explicación proposicional del significado, el conjunto de los significados que un texto normativo puede asumir es contingente y a priori $^{33}$. Los miembros de este conjunto son en efecto definiciones lexicales generales y abstractas, las cuales habrían podido ser diferentes de aquellas que de hecho son, en el caso que hubiesen sido diferentes las reglas del lenguaje, los cánones interpretativos y las distinciones dogmáticas aceptadas. Los miembros de este conjunto, como también el "marco" que los contiene, son por lo tanto contingentes. Además, los significados admisibles en el tiempo t0, pueden ser conocidos antes que la interpretación tenga lugar en el tiempo t1, eso consiente que el intérprete los conozca antes de comenzar a interpretar el texto normativo y le permite elegir uno de ellos con fines decisorios. Bajo esta perspectiva, los significados admisibles son por lo tanto cognoscibles a priori, el marco circunscribe los significados que un enunciado normativo puede asumir en t0 y que todavía no ha asumido en t1. Estando así las cosas, tiene perfectamente sentido distinguir entre la interpretación y la integración del derecho. Esta última consiste en elegir para $\mathrm{T}$ en $\mathrm{t} 1$ un significado no incluido entre aquellos admisibles en t0. En base a esta reconstrucción se evitan los resultados contraintuitivos a los que, en cambio, conduce el escepticismo radical.

Esta tesis, sin embargo, oscurece una circunstancia muy relevante, el hecho que los intérpretes modifican diacrónicamente los contenidos del derecho atribuyendo a los textos normativos contenidos nuevos, mediante la denominada interpretación evolutiva. Desde la perspectiva que estamos describiendo, estos cambios son obviamente explicados como una consecuencia de cambios en las reglas del lenguaje, en los cánones interpretativos aceptados y en las tesis dogmáticas utilizadas por los intérpretes. Desde el punto de vista lógico, sin embargo, el

${ }^{33}$ Los términos "contingente", "necesario", "a priori" y "a posteriori" serán a continuación utilizados en la acepción propuesta por Saul Kripke en Naming and Necessity, Cambridge (MA), Harvard University Press, 1980, Lesson 1. En el léxico de Kripke, "contingente" y "necesario" son términos que expresan conceptos ontológicos, mientras "a priori" y "a posteriori" son términos que expresan conceptos epistémicos. 
cambio de los criterios interpretativos apenas catalogados no puede convertirse en actual, y ser por lo tanto cognoscible, antes que una interpretación decisoria tenga lugar, sino solamente a través o en virtud de tal interpretación. Ni tampoco puede valer la tesis según la cual la modificación de los significados normativos se realiza por medio de la legislación intersticial. Admitamos en efecto que el juez G1 atribuye el significado $\mathrm{S}$ a $\mathrm{T}$ en $\mathrm{t} 1$ y que en $\mathrm{t} 1 \mathrm{~S}$ no sea uno de los significados admisibles de T, puesto que se sitúa fuera del marco. Supongamos además que también el juez $\mathrm{G} 2$ atribuye $\mathrm{S}$ a $\mathrm{T}$ en un tiempo sucesivo a t2, y que lo mismo haga G3 en t3, G4 en t4...Gn en tn. A un cierto punto, se dirá, S ha comenzado a formar parte de los significados admisibles de $\mathrm{T}$, la extensión del marco ha sido a tal punto modificada que ahora lo contiene. Podemos preguntarnos, sin embargo, ¿en qué momento esto ha sucedido? No se trata de una pregunta ociosa, porque una concepción contingente y a priori del marco presupone que los significados admisibles sean cognoscibles antes que la interpretación tenga lugar. Ahora, ciertamente tal cambio no puede tener lugar en $\mathrm{t} 1$. Si un acto de legislación intersticial tuviese como tal el poder de modificar el marco de los significados admisibles, incluso cuando se coloca fuera del marco, la distinción entre interpretación e integración del derecho desaparecería, puesto que cualquier atribución de significado podría delinear un marco capaz de contenerla ${ }^{34}$. Pero este cambio no puede tampoco tener lugar en tn, si fuera así, en efecto, el marco no sería determinable a priori sino solamente a posteriori, luego que $\mathrm{T}$ haya sido interpretado; circunstancia ésta que volvería la teoría autocontradictoria. En el intervalo entre $\mathrm{t} 2 \mathrm{y}$ tn, finalmente, el intérprete no es capaz de conocer si $\mathrm{S}$ es uno de los significados admisibles de $\mathrm{T}$. En este lapso temporal, en efecto, la extensión del marco está todavía indeterminada respecto de $\mathrm{S}$, no está claro si $\mathrm{S}$ es o no uno de los significados que $\mathrm{T}$ puede asumir en base a las reglas del lenguaje, a los cánones interpretativos y a las tesis dogmáticas aceptadas. En el

${ }^{34} \mathrm{He}$ desarrollado ulteriormente este argumento en D. Canale, Paradossi della consuetudine giuridica, en S. Zorzetto (ed.), La consuetudine giuridica: teoria, storia, ambiti disciplinari, Pisa, ETS, 2010, pp. 109-136. 
intervalo entre $\mathrm{t} 2 \mathrm{y} \mathrm{tn}$, por lo tanto, no es posible conocer si $\mathrm{S}$ es un significado atribuible a T. A la luz de estas consideraciones, resulta evidente que una concepción contingente y a priori del marco genera una paradoja, que podríamos denominar "paradoja de la inmutabilidad de los significados admisibles". Se trata de una paradoja que quien acepta esta tesis tiene la carga de resolver.

Si adoptamos en cambio una explicación interpretativa del significado, el conjunto de los significados que un texto normativo puede asumir es cognoscible solamente a posteriori. Este conjunto está en efecto constituido por definiciones estipulativas particulares y concretas, a veces simplemente repetitivas de definiciones estipulativas precedentes, pero que de todos modos son una condición necesaria y suficiente para que un término o una expresión lingüística signifiquen algo. El conjunto de estas definiciones no podrá sin embargo ser conocido antes que la interpretación decisoria tenga lugar, porque el acto interpretativo coincide con el acto definitorio, o sea, con el acto que fija el significado posible o admisible.

El marco de los significados admisibles en t1 contendrá por lo tanto los significados que, de hecho, un enunciado normativo ha asumido hasta $\mathrm{t} 1$-y que por lo tanto, a fuerza del principio de Leibniz ${ }^{35}$, podia asumir hasta aquel momento- sin que eso excluya que el mismo enunciado pueda asumir en $\mathrm{t} 2$ un significado que en $\mathrm{t} 1$ se situaba fuera del marco. Se sigue que, tanto el estudioso del derecho como el juez, no pueden distinguir una instancia de interpretación decisoria de una instancia de legislación intersticial, antes que una elección interpretativa haya sido concretamente cumplida en sede decisoria. Por lo que el marco de los significados admisibles podrá ser trazado solamente ex post, después que un "significado" haya sido atribuido al texto normativo interpretado ${ }^{36}$.

${ }^{35} \mathrm{Me}$ refiero al principio de razón suficiente, en base al cual nada sucede en el mundo sin que exista la posibilidad de que suceda y sin que exista por lo tanto una razón o causa que lo explique.

${ }^{36}$ Nos podríamos preguntar además, ¿el marco de los significados admisibles a posteriori es necesario o contingente? Con relación a este problema de tipo 
Esto no equivale a sostener -nótese bien- que los textos normativos no posean significados estándares y que puedan por eso asumir un significado cualquiera, sobre la base de las elecciones idiosincráticas del intérprete, tal como sostiene la teoría escéptico-radical de la interpretación. Lo apenas dicho evidencia simplemente la equivocidad de la noción de marco y, por lo tanto, la noción de significado estándar. En el contexto de una concepción proposicional del significado, "significado estándar" es una noción semántica que denota una relación entre los enunciados y el mundo, noción de la cual depende la noción de interpretación. En el contexto de una concepción interpretativa del significado, en cambio, "significado estándar" es una noción pragmática que presupone la noción de interpretación y que denota el comportamiento de los hablantes orientado a volver previsibles los efectos de una proferencia, a economizar los recursos necesarios para la comunicación ${ }^{37}$, o bien a perseguir finalidades de tipo ético y político. Retomando el léxico de Tarello y Guastini, el significado estándar constituye, desde esta perspectiva, la expresión de una ideología de la interpretación.

Por lo tanto, no solo es perfectamente sensato hablar de una concepción interpretativa de los significados estándares y del marco que los contiene, si no que además tal concepción tiene la ventaja, respecto a aquella proposicional, de explicar el hecho que los significados jurídicos cambian en el tiempo gracias a la interpretación y no a pesar de ella. Tal concepción, sin embargo, vuelve poco claro otro hecho, no

ontológico, basta observar aquí lo siguiente. En el caso que las normas jurídicas consistan en hechos institucionales que supervienen a hechos empíricos, o que incluso son independientes de ellos, los significados admisibles a posteriori de un enunciado normativo son contingentes, desde el momento que podrían haber sido diferentes de los que de hecho son hasta este momento. En el caso que, en cambio, las normas jurídicas sean consideradas reducibles, sin residuo alguno, a hechos empíricos susceptibles de ser explicados utilizado las leyes de la física, entonces los significados admisibles a posteriori son necesarios, puesto que dadas las leyes de la física, tales significados no habrían podido ser diferentes de aquellos que de hecho son hasta este momento.

${ }^{37}$ Véase Davidson, Radical Interpretation, cit. 
Teorías de la interpretación jurídica....

menos relevante que el primero. Se trata de la circunstancia que los significados normativos en ámbito jurídico son altamente estandarizados, una estandarización que en muchos ámbitos del derecho es muy extendida. Esta estandarización prescinde, aparentemente, de la variedad de inclinaciones individuales y de la pluralidad de convicciones políticas y morales que caracterizan a los intérpretes jurídicos ${ }^{38}$. La explicación de este hecho ofrecida por una teoría "interpretativa" de la interpretación jurídica es, a decir la verdad, muy poco convincente.

\section{Conclusión}

Si cuanto hemos observado hasta aquí es acertado, Guastini es llamado a resolver las ambigüedades que afligen a la teoría escépticomoderada de la interpretación, precisando cuál es la explicación general del funcionamiento del lenguaje que ella presupone y derivando a partir de ella las debidas consecuencias relativas a los contenidos y a las implicaciones de la misma teoría.

Se podría sin embargo objetar, llegados a este punto, que el teórico de la interpretación no está obligado a tomar en serio estos pedidos. Su tarea consiste simplemente en contribuir al conocimiento de un hecho social, las prácticas interpretativas, construyendo un modelo capaz de dar cuenta de sus características más importantes. Este modelo está compuesto por un conjunto de principios o axiomas -que incluyen, en nuestro caso, una definición estipulativa del término "interpretación" y la distinción entre texto normativo (disposición) y significado normativo (norma)- a partir de los cuales se derivan, recurriendo a reglas de transformación de tipo lógico y de tipo semántico, las tesis de la teoría, que describen las características importantes de su objeto de investigación. Si la representación de los enunciados interpretativos ofrecida por estas afirmaciones, se corresponde con los enunciados interpretativos realmente proferidos por los jueces y por los estudiosos

38 Véase sobre este punto B. Leiter, Explaining Theoretical Disagreements, "University of Chicago Law Review", 76, 2009, pp. 1215 y ss 
del derecho, de modo que los principios de la teoría adquieren, parafraseando a Hempel, un "contenido empírico"39, entonces la teoría ha alcanzado su objetivo descriptivo y no necesita ulteriores especificaciones. Cualquier referencia a las theories of meaning mencionadas anteriormente se reduciría, al fin de cuentas, a la formulación de hipótesis de tipo psicológico, relativas al funcionamiento de los procesos mentales de los intérpretes en el curso de la interpretación-proceso. Se trata de una cuestión que merece ciertamente la atención de la psicología cognitiva, pero no la del teórico del derecho, cuya tarea no es investigar el funcionamiento de la mente sino describir, explicar o prever los hechos sociales.

Esta objeción es de gran interés porque se refiere al núcleo de las características y de las tareas de una teoría de la interpretación jurídica. A ella están dedicadas las dos observaciones conclusivas de esta intervención.

En primer lugar, la idea que una meaning theory sea un discurso psicológico, que da una representación del funcionamiento de la mente del intérprete, es equivocada. Las teorías del significado de tipo proposicional e interpretativo son discursos filosóficos que intentan, al contrario, colmar el "gap explicativo" que aflige a toda explicación fisicalista de la competencia lingüística y de la comunicación ${ }^{40}$. Las neurociencias pueden ciertamente permitirnos registrar la actividad bioquímica del cerebro y la psicología cognitiva puede asociar a esta actividad estados mentales subjetivos; no obstante, estas investigaciones no son capaces de describir y explicar la experiencia por la que pasa un ser humano cuando comprende una expresión lingüística y cuando comunica su contenido. Una theory of meaning intenta colmar este gap

${ }^{39}$ C.G. Hempel, On the Structure of Scientific Theories, en The Philosophy of Carl G. Hempel. Studies in Science, Explanation, and Rationality, Oxford, Oxford University Press, 2001, p. 50.

${ }^{40}$ Véase, por último, D.J. Chalmers, Phenomenal Concepts and the Explanatory Gap, en T. Alter-S. Walter (eds.), Phenomelan Concepts and Phenomenal Knowledge: New Essays on Consciousness and Physicalism, Oxford, Oxford University Press, 2007, pp. 167-194. Véase también S. Blackburn, Spreading the Word. Groundings in the Philosophy of Language, Oxford, Clarendon Press, 1984, pp. 60 y ss. 
recurriendo al análisis conceptual además de la observación empírica de los comportamientos lingüísticos. Bajo este aspecto, su discurso, por lo tanto, no es asimilable al de la psicología cognitiva y sirve en cambio de base a la teoría de la interpretación, con la cual comparte la misma "caja de herramientas" teóricas.

En segundo lugar, una teoría de la interpretación jurídica no surge de la nada, sino que constituye la respuesta a uno o más problemas que el estudioso del derecho considera relevantes e interesantes en el ámbito de su investigación. Tales problemas desarrollan un papel muy importante en la construcción de la teoría, porque de estos depende la formulación de los principios o axiomas de los que la nueva teoría parte, así como también la selección de los aspectos de las prácticas interpretativas que merecen atención. En el caso de la teoría escéptico-moderada de la interpretación, los problemas en juego me parecen esencialmente dos: "¿qué relación existe entre legislación y jurisdicción, o sea entre la producción de las disposiciones jurídicas y su interpretación en sede decisoria?"; y "si el resultado de la interpretación decisoria es discrecional, ¿esta discrecionalidad está sometida a límites?". Si entendida como una respuesta a estos dos problemas, una teoría de la interpretación constituye un discurso preliminar de la teoría del ordenamiento del Estado y permite desmitificar, bajo la perspectiva defendida por Guastini, los puntos de vista de tipo ideológico que niegan o radicalizan el carácter discrecional de las decisiones de las cortes. Sin embargo, es legítimo preguntarse si son solo éstas las preguntas a las cuales una teoría de la interpretación debe buscar una respuesta, o si existen otras, en verdad no menos relevantes e interesantes, a las cuales las teorías tradicionales de la interpretación no ofrecen respuesta alguna. Creo que reconocer la relevancia para una teoría de la interpretación jurídica del problema filosófico del significado, más allá de volver más sólidas sus bases filosóficas, puede contribuir a ampliar el campo de investigación hacia aspectos de la praxis interpretativa, generalmente no tomados en consideración por los teóricos del derecho y que son no obstante de gran interés para los juristas. Esta apertura de perspectiva no perjudicaría el rigor que caracteriza a una teoría de la interpretación como aquella elaborada por Riccardo Guastini, pero volvería más ricos y fecundos los contenidos, consintiendo desarrollos ulteriores. 